\title{
Novel Distributed PZT Active Vibration Control Based on Characteristic Model for the Space Frame Structure
}

\author{
Hua Zhong, ${ }^{1}$ Yong Wang, ${ }^{2}$ Hanzheng Ran, ${ }^{1}$ Qing Wang, ${ }^{1}$ and Changxing Shao ${ }^{2}$ \\ ${ }^{1}$ Institute of Electronic Engineering, China Academy of Engineering Physics, Mianyang 621999, China \\ ${ }^{2}$ Department of Automation, University of Science and Technology of China, Hefei 230027, China \\ Correspondence should be addressed to Hua Zhong; huazhong@mail.ustc.edu.cn
}

Received 6 July 2015; Revised 19 September 2015; Accepted 21 September 2015

Academic Editor: Mario Terzo

Copyright (c) 2016 Hua Zhong et al. This is an open access article distributed under the Creative Commons Attribution License, which permits unrestricted use, distribution, and reproduction in any medium, provided the original work is properly cited.

\begin{abstract}
A novel distributed PZT control strategy based on characteristic model is presented for space frame structure in this paper. It is a challenge to obtain the exact mechanical model for space structure, since it is a coupling MIMO plant with unknown parameters and disturbances. Thus the characteristic modeling theory is adopted to establish the needed model, which can accurately describe the dynamic characteristics of the space frame structure in real time. On basis of this model, a keep tracking controller is designed to suppress the vibration actively. It is shown that the proposed model-free method is very robust and easy to implement. To solve the complex and difficulty problem on PZT location optimization, an efficient method with modal strain energy and maximum vibration amplitude is proposed. Finally, a simulation study is conducted to investigate the effectiveness of the proposed active vibration control scheme.
\end{abstract}

\section{Introduction}

Space structures are usually applied in the aerospace and civil engineering such as the space boom structures [1]. Generally, since space structures are light and flexible, they are sensitive to vibration which will affect the performance of space equipment [2]. Therefore, it is necessary to suppress vibration for space structure.

Active vibration control method is a good choice for the vibration control of space structure. To achieve good vibration control performance, there are three things to be done, that is, system modelling, sensors/actuators location configuration, and control strategy design.

Space structure's model can be divided into two categories: mechanical model and identification model. The mechanical model is usually established with sophisticated finite element method [3,4]. Additionally, it is generally used in system dynamics analysis and sensors/actuators location optimization. Nonetheless, it can not be used to design controller directly due to its complexity and inaccuracy. Due to the tremendous efforts devoted by researchers, valuable results on identification modeling for the space structure have been obtained. Hwang presented an analytical procedure based on the Kalman filtering approach to estimate modal loads applied on a structure [5]. Hazra et al. proposed a method which integrates the blind identification with timefrequency decomposition of signals for flexible structures [6]. Schoen et al. presented an identification algorithm which utilizes modal contribution coefficients to monitor the data collection for large flexible space structures [7]. Although some aforementioned methods have been extensively used in space structure identification, there still exist several disadvantages. On the one hand, the system is inherently complicated, much less the disturbances. On the other hand, the existing results only depend on complex off-line identification methods, which is difficult to grasp the system dynamics in real time. Consequently, it is a challenge to establish the model for space structure with on-line identification method.

For active vibration control of space structure, determination of actuator locations is an important work. If locations of actuators are not proper, active vibration control may not suppress the vibration, even it can increase structure vibration. Therefore, optimization of actuators' locations has attracted many scholars to study. Chen et al. presented 
a location optimization approach based on the 1st singular value perturbations of observability and controllability [8]. $\mathrm{Xu}$ and Jiang demonstrated an approach with the controllability and observability index of the system [4]. Zhao et al. proposed a continuous variable optimization method to solve the optimal placement of piezoelectric active bars [2]. Li and Huang presented a layered optimization strategy to address location optimization problem [9]. Though the above methods can realize location optimization actuators, the optimization process is complex. Thus, it is needed to find a simple and effective optimization method.

An active vibration controller with simple structure and strong robustness is necessary. Many scholars have devoted themselves to solving this problem. Luo et al. designed simple PD controller for vibration attenuation in Hoop truss structure [10]. Abreu et al. used a standard $H_{\infty}$ robust controller to suppress structural vibrations [11]. Yang et al. presented an adaptive fuzzy sliding mode controller for vibration of a flexible rectangular plate [12]. Lin and Zheng proposed a parallel neurofuzzy control with genetic algorithm tuning for smart piezoelectric rotating truss structure [13]. de Abreu et al. designed a self-organizing fuzzy controller for vibration control of smart truss structure [1]. Mahmoodi developed a modified positive position feedback controller for vibration control [14]. Wilhelm and Rajamani applied LQR controller to realize multimodal vibration suppression [15]. Among above control strategies, simple controllers (such as PID) are with weak robustness. The complex controllers $\left(H_{\infty}\right.$ controller, fuzzy controller) have strong robustness, but they have difficulty in parameter tuning.

Motivated by the above discussions, a novel distributed PZT control based on characteristic model is presented. Characteristic model for space structure is established to accurately describe system's dynamics in time whose form is simple and characteristic parameters of it can contain the information of time delay or high order [16]. Additionally, a simple controller based on characteristic model called keep tracking controller with strong robustness is applied to realize vibration control. Furthermore, a novel actuators location optimization method with modal strain energy (MSE) and maximum vibration amplitude is proposed.

The remainder of the paper is organized as follows. Section 2 establishes the characteristic model for space structure. Section 3 presents the identification of characteristic parameters and control strategy. PZT location optimization is presented in Section 4. Numerical simulation is shown in Section 5. Conclusion is shown in Section 6.

\section{Characteristic Modeling for Space Structure}

The space frame structure is shown in Figure 1 which contains 26 nodes and 63 beam elements. Nodes 2, 3, 7, and 8 are constrained which are fixed with the floor. Structure excitation direction is $z$ of node 10. Every node has 6 degrees of freedom (DOF). The total DOF is $n$. The space structure has 63 beam elements. Table 1 shows the connection information among nodes. Space structure consists of aluminum whose cross section is rectangular. Table 2 shows the top 10 natural frequencies.

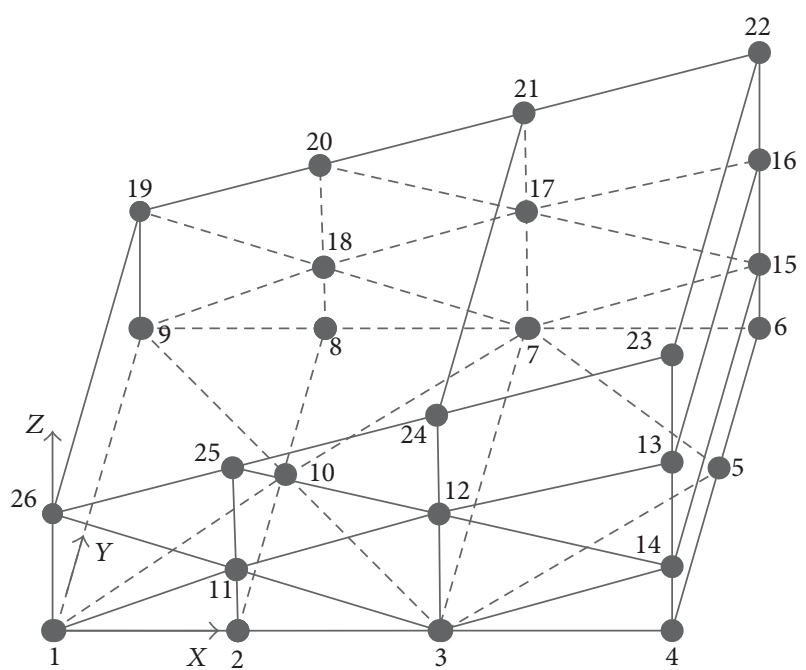

FIGURE 1: The schematic diagram of frame structure.

According to the finite element formulation, the equation of motion for any structure is given by

$$
\mathbf{M X}+\mathbf{C} \dot{\mathbf{X}}+\mathbf{K X}=\mathbf{F},
$$

where $\mathbf{M} \in \mathbf{R}^{n \times n}$ is mass matrix, $\mathbf{C} \in \mathbf{R}^{n \times n}$ is damping matrix, $\mathbf{K} \in \mathbf{R}^{n \times n}$ is stiffness matrix, $\mathbf{F} \in \mathbf{R}^{n \times 1}$ is load force vector, and $\mathbf{X} \in \mathbf{R}^{n \times 1}, \dot{\mathbf{X}} \in \mathbf{R}^{n \times 1}$, and $\ddot{\mathbf{X}} \in \mathbf{R}^{n \times 1}$ are structural displacement, velocity, and acceleration vectors, respectively.

With the knowledge of space frame, parameters $\mathbf{M}, \mathbf{C}$, and $\mathbf{K}$ are slow time-varying. Then, during short sampling time $\Delta t(\mathrm{~ms})$, it can be considered that $\mathbf{M}, \mathbf{C}$, and $\mathbf{K}$ are timeinvariant. Then the discrete form of motion equation (1) can be described as

$$
\begin{aligned}
\mathbf{M X}(k+1)= & \left(2 \mathbf{M}-\mathbf{C} \Delta t-\mathbf{K} \Delta t^{2}\right) \mathbf{X}(k) \\
& +(-\mathbf{M}+\mathbf{C} \Delta t) \mathbf{X}(k-1)+\Delta t^{2} \mathbf{F}(k)
\end{aligned}
$$

One has the upper equation (2) as

$$
\begin{aligned}
\mathbf{X}(k+1)= & \mathbf{G}_{1}(k) \mathbf{X}(k)+\mathbf{G}_{2}(k) \mathbf{X}(k-1) \\
& +\mathbf{H}_{1}(k) \mathbf{F}(k),
\end{aligned}
$$

where $\mathbf{G}_{1}(k)=2 \mathbf{I}_{n}-\mathbf{M}^{-1} \mathbf{C} \Delta t-\mathbf{M}^{-1} \mathbf{K} \Delta t^{2}, \mathbf{G}_{2}(k)=$ $-\mathbf{I}_{n}+\mathbf{M}^{-1} \mathbf{C} \Delta t$, and $\mathbf{H}_{1}(k)=\Delta t^{2} \mathbf{M}^{-1}$. The expression (3) is called characteristic model; $\mathbf{G}_{1}(k), \mathbf{G}_{2}(k)$, and $\mathbf{H}_{1}(k)$ are characteristic parameters.

When $\Delta t$ is very small, the following equation holds:

$$
\begin{aligned}
& \mathbf{G}_{1}(\infty)=2 \mathbf{I}_{n}, \\
& \mathbf{G}_{2}(\infty)=-\mathbf{I}_{n}, \\
& \mathbf{H}_{1}(\infty)=\mathbf{0}_{n \times n} .
\end{aligned}
$$

Further one has the sum of characteristic parameters as

$$
\mathbf{G}_{1}(\infty)+\mathbf{G}_{2}(\infty)+\mathbf{H}_{1}(\infty)=\mathbf{I}_{n} .
$$


TABLE 1: Beam element connection information.

\begin{tabular}{|c|c|}
\hline Beam number & Node number \\
\hline 1 & $1-2$ \\
\hline 2 & $2-3$ \\
\hline 3 & $3-4$ \\
\hline 4 & $4-5$ \\
\hline 5 & $5-6$ \\
\hline 6 & $6-7$ \\
\hline 7 & $7-8$ \\
\hline 8 & $8-9$ \\
\hline 9 & $9-1$ \\
\hline 10 & $9-10$ \\
\hline 11 & $1-10$ \\
\hline 12 & $2-10$ \\
\hline 13 & $10-8$ \\
\hline 14 & $10-7$ \\
\hline 15 & $10-3$ \\
\hline 16 & $3-7$ \\
\hline 17 & $3-5$ \\
\hline 18 & $5-7$ \\
\hline 19 & $1-26$ \\
\hline 20 & $25-11$ \\
\hline 21 & $11-2$ \\
\hline 22 & $24-12$ \\
\hline 23 & $12-3$ \\
\hline 24 & $23-13$ \\
\hline 25 & $13-14$ \\
\hline 26 & $14-4$ \\
\hline 27 & $15-6$ \\
\hline 28 & $16-15$ \\
\hline 29 & $22-16$ \\
\hline 30 & $17-7$ \\
\hline 31 & $21-17$ \\
\hline 32 & $18-8$ \\
\hline 33 & $20-18$ \\
\hline 34 & $19-9$ \\
\hline 35 & $19-26$ \\
\hline 36 & $26-25$ \\
\hline 37 & $26-11$ \\
\hline 38 & $1-11$ \\
\hline 39 & $25-24$ \\
\hline 40 & $25-12$ \\
\hline 41 & $11-12$ \\
\hline 42 & $11-3$ \\
\hline 43 & $24-23$ \\
\hline 44 & $24-13$ \\
\hline 45 & $12-13$ \\
\hline 46 & $12-14$ \\
\hline 47 & $3-14$ \\
\hline 48 & $23-22$ \\
\hline 49 & $13-16$ \\
\hline
\end{tabular}

TABLE 1: Continued.

\begin{tabular}{lc}
\hline Beam number & Node number \\
\hline 50 & $14-15$ \\
51 & $21-22$ \\
52 & $21-16$ \\
53 & $17-16$ \\
54 & $17-15$ \\
55 & $7-15$ \\
56 & $20-21$ \\
57 & $20-17$ \\
58 & $18-17$ \\
59 & $18-7$ \\
60 & $19-20$ \\
61 & $19-18$ \\
62 & $9-18$ \\
63 & $24-21$ \\
\hline
\end{tabular}

Assuming the output $\mathbf{X}(k)$ loops are decoupled, thus $\mathbf{G}_{1}(k)$ and $\mathbf{G}_{2}(k)$ are diagonal matrixes, which can be formulated as

$$
\begin{aligned}
& \mathbf{G}_{1}(k)=\left[\begin{array}{cccc}
g_{11}(k) & 0 & \cdots & 0 \\
0 & g_{12}(k) & \cdots & 0 \\
\vdots & \vdots & \ddots & \vdots \\
0 & 0 & \cdots & g_{1 n}(k)
\end{array}\right], \\
& \mathbf{G}_{2}(k)=\left[\begin{array}{cccc}
g_{21}(k) & 0 & \cdots & 0 \\
0 & g_{22}(k) & \cdots & \vdots \\
\vdots & \vdots & \ddots & 0 \\
0 & 0 & \cdots & g_{2 n}(k)
\end{array}\right] .
\end{aligned}
$$

The characteristic parameter $\mathbf{H}_{1}(k)$ can be written as

$$
\mathbf{H}_{1}(k)=\left[\begin{array}{cccc}
h_{11}(k) & h_{12}(k) & \cdots & h_{1 n}(k) \\
h_{21}(k) & h_{22}(k) & \cdots & h_{2 n}(k) \\
\vdots & \vdots & \ddots & \vdots \\
h_{n 1}(k) & h_{n 2}(k) & \cdots & h_{n n}(k)
\end{array}\right]
$$

According to the expression in (3) and (6)-(7), the ith characteristic model is

$$
\begin{aligned}
x_{i}(k+1)= & g_{1 i}(k) x_{i}(k)+g_{2 i}(k) x_{i}(k-1) \\
& +\sum_{r=1}^{n} h_{i r}(k) F_{r}(k) .
\end{aligned}
$$


TABLE 2: Top 10 natural frequencies.

\begin{tabular}{lcccccccccc}
\hline Order & 1 & 2 & 3 & 4 & 5 & 6 & 7 & 8 & 9 & 10 \\
\hline Frequency $(\mathrm{Hz})$ & 10.03 & 15.51 & 18.02 & 22.82 & 25.85 & 27.72 & 29.35 & 31.45 & 42.22 & 42.61 \\
\hline
\end{tabular}

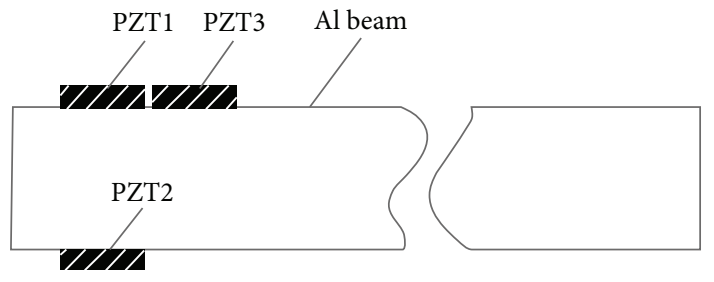

FIgURE 2: The schematic diagram of PZT structure.

Similarly, one has the characters of characteristic parameters in $i$ th output loop as

$$
\begin{aligned}
g_{1 i}(\infty) & =2, \\
g_{2 i}(\infty) & =-1 \\
h_{i r}(\infty) & =0 \\
g_{1 i}(\infty)+g_{2 i}(\infty)+\sum_{r=1}^{n} h_{i r}(\infty) & =1
\end{aligned}
$$

Because PZT with small size and light weight does not affect structure's characteristics after pasting on the structure surface, PZT is selected as sensor and actuator. Figure 2 indicates the schematic diagram of PZT structure. It is shown that a pair of PZT1 and PZT2 act as actuator and another PZT3 acts as sensor. Additionally, actuators far away PZT3 have little function on PZT3. Therefore, (8) can be simplified as

$$
\begin{aligned}
x_{i}(k+1)= & g_{1 i}(k) x_{i}(k)+g_{2 i}(k) x_{i}(k-1) \\
& +h_{i i}(k) F_{i}(k)=\boldsymbol{\Phi}^{T}(k) \boldsymbol{\vartheta}(k),
\end{aligned}
$$

where $\boldsymbol{\Phi}(k)=\left[x_{i}(k) x_{i}(k-1) F_{i}(k)\right]^{T}$ and $\boldsymbol{\vartheta}(k)=$ $\left[\begin{array}{lll}g_{1 i}(k) & g_{2 i}(k-1) & h_{i i}(k)\end{array}\right]^{T}$.

\section{Keep Tracking Control Law}

With a proper parameter estimator, the characteristic parameters can adaptively converge to true values. The general methods of parameters identification are least square method and gradient method. Compared with the least square method, gradient method has a smaller amount of calculation and it will not cause parameters to diverge with improper forgetting factors. Thus, the gradient method [16] will be used to estimate the characteristic parameters. The form is

$$
\begin{aligned}
\widehat{\boldsymbol{\vartheta}}(k)= & \frac{\lambda_{1} \Phi(k-1)\left[x_{i}(k)-\Phi^{T}(k-1) \widehat{\boldsymbol{\vartheta}}(k-1)\right]}{\boldsymbol{\Phi}^{T}(k-1) \Phi(k-1)+\lambda_{2}} \\
& +\widehat{\boldsymbol{\vartheta}}(k-1) .
\end{aligned}
$$

The range of $\lambda_{1}$ and $\lambda_{2}$ is $0<\lambda_{1}<1,0<\lambda_{2}<4$. The estimates of $g_{1 i}(k), g_{2 i}(k)$, and $h_{i i}(k)$ in the characteristic model are $\widehat{\boldsymbol{\vartheta}}^{T}(k)=\left[\begin{array}{lll}\widehat{g}_{1 i}(k) & \widehat{g}_{2 i}(k) & \widehat{h}_{i i}(k)\end{array}\right]$.

To ensure the output $y(k+1)$ equals the desired reference input $r(k+1)$, the need control input should satisfy the following condition:

$$
u(k)=\frac{r(k+1)-\widehat{g}_{1 i}(k) y(k)-\widehat{g}_{2 i}(k) y(k-1)}{\widehat{h}_{i i}(k)} .
$$

To avoid the fact of $\widehat{h}_{i i}(0)=0$, a slack variable $\lambda$ is introduced, which could guarantee that the control input is smooth and easy to implement. Consider

$$
u(k)=\frac{r(k+1)-\widehat{g}_{1 i}(k) y(k)-\widehat{g}_{2 i}(k) y(k-1)}{\lambda+\widehat{h}_{i i}(k)},
$$

where $\lambda$ is a constant. This control law is called characteristic model-based keep tracking control (CMKTC) law [16, 17]. With the control law, system can perfectly track the reference input.

\section{PZT Location Optimization}

Considering the complexity of traditional optimization algorithm, MSE and maximum amplitude are applied to optimize locations of PZT whose optimization process is simple.

Firstly beam elements of space structure are divided into three categories: underside beam elements $N_{u}=$ $\{1,2, \ldots, 17,18\}$, front and rear beam elements $N_{f r}=$ $\{19,20, \ldots, 33,34,36,37, \ldots, 46,47,51,52, \ldots, 61,62\}$, and connection beam elements between front and rear $N_{c}=\{35,48,49,50,63\}$. Through beams' MSE, the sensitive beams are obtained. With ith natural frequency, the $j$ th beam's MSE [18] can be described as

$$
E_{i j}=\frac{\phi_{i}^{T} \mathbf{K}_{j} \phi_{i}}{\omega_{i}^{2}},
$$

where $\phi_{i}$ is modal shape vector with $i$ th natural frequency which is the ith column of $\mathbf{V}_{\text {norm }} \cdot \mathbf{V}_{\text {norm }}=\mathbf{V}\left(\sqrt{\mathbf{V}^{T} \mathbf{M} V}\right)^{-1}$ is normalized modal matrix, where $V$ is modal matrix. $\mathbf{K}_{j}$ is $j$ th beam stiffness matrix; $\omega_{i}$ is $i$ th vibration frequency.

Figures 3-6 show the first four order MSE. From the figures, sensitive beam element sets are obtained: underside sensitive beam element set $N_{s}=\{1,4,5,9,10,11\}$, front and rear sensitive beam element set $N_{b}=$ $\{19,20,23,25,28,34,36,37\}$, and connection sensitive beam element set $N_{h}=\{35,48,49,50,63\} . N_{s}, N_{b}$, and $N_{h}$ are the subsets of $N_{u}, N_{f r}$, and $N_{c}$, respectively.

Additionally, it is needed to select the most sensitive beam element from $N_{s}, N_{b}$, and $N_{h}$ according to maximum 
TABLE 3: $z$-direction maximum amplitude of beams in $N_{s}$.

\begin{tabular}{lcccccc}
\hline Beam number & 1 & 4 & 5 & 9 & 10 & 11 \\
\hline Maximum amplitude $(\mathrm{mm})$ & 0.0005 & 0.0013 & 0.0015 & 0.1422 & 0.3996 & 0.3996 \\
\hline
\end{tabular}
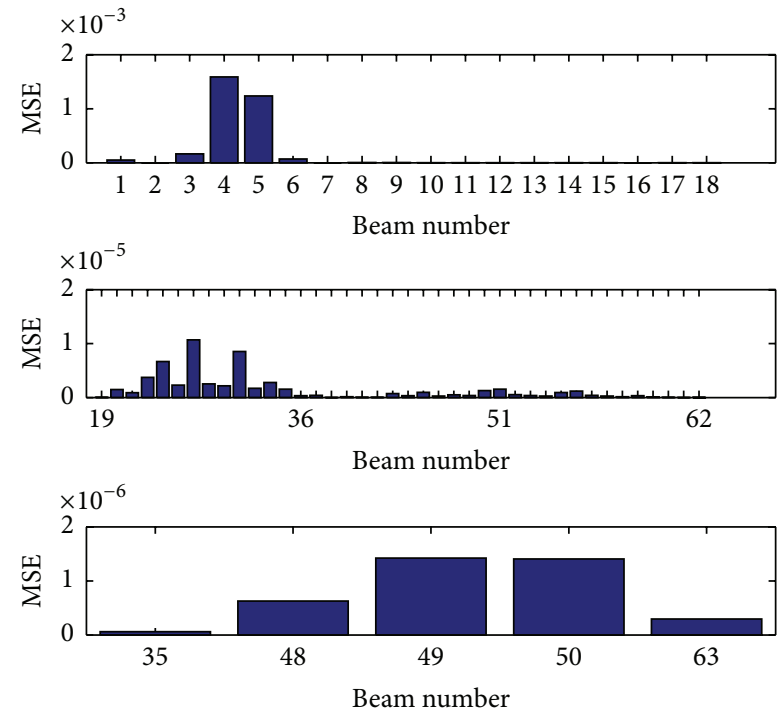

FIGURE 3: First-order MSE.
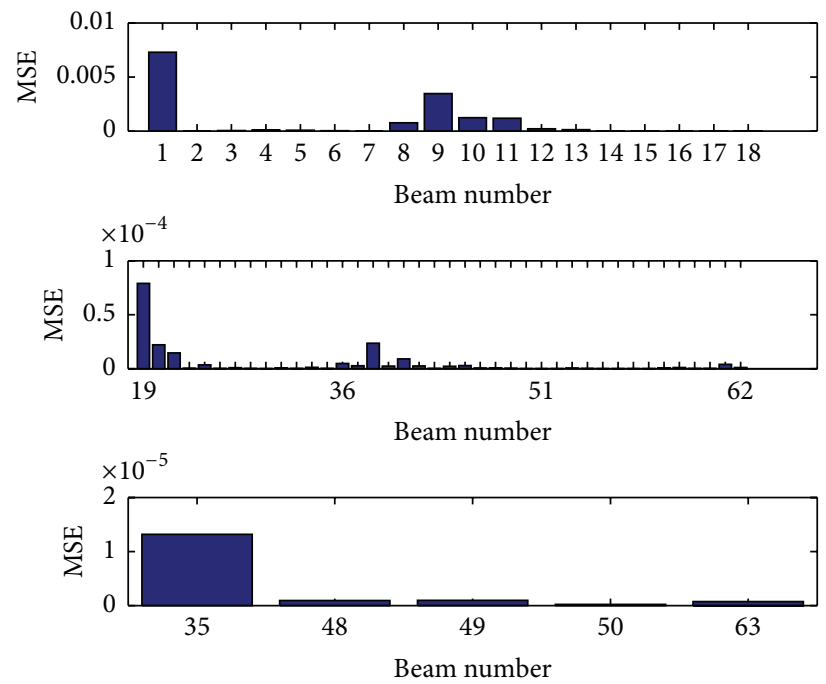

Figure 4: Second-order MSE.

amplitude. Excitation node is selected as node 10 and excitation direction is $z$. Excitation force $f_{e}=10 \sin (2 \pi f t)$, and excitation frequency $f$ is selected as the first natural frequency $10.03 \mathrm{~Hz}$. Through analysing vibration response curves of $N_{s}$, it is concluded that $z$-direction vibration amplitude is much larger than $x$ and $y$ which is considered. Similarly, analysing vibration response curves of $N_{b}$ and $N_{h}$, it is shown that $y$-direction vibration amplitude is much larger than $x$ and $y$. Thus, it is only needed to compare $z$-direction of the maximum amplitude for beams in $N_{b}$ and $N_{h}$.

From Table 3 which shows the $z$-direction maximum vibration amplitude of $N_{s}$, 10th or 11th beam element is
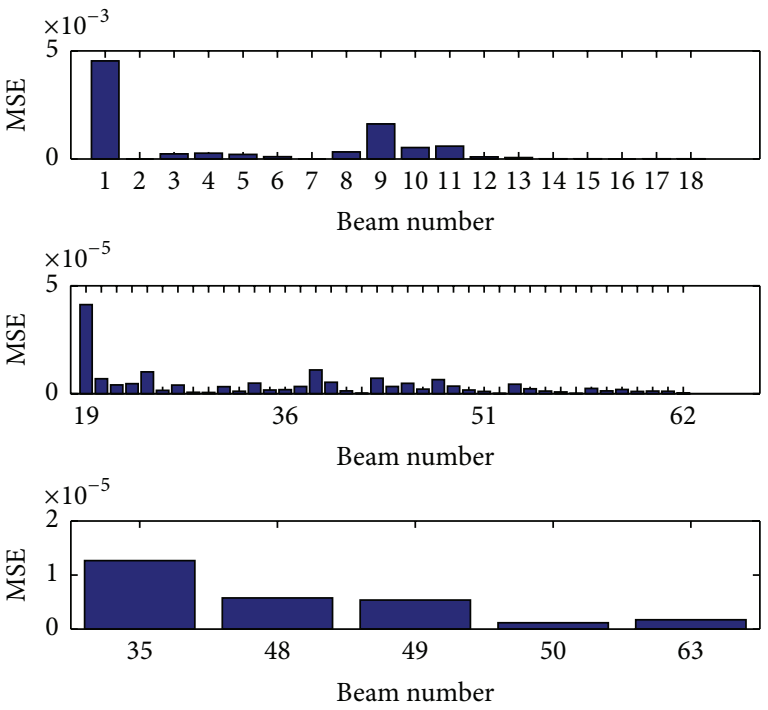

FIgURE 5: Third-order MSE.
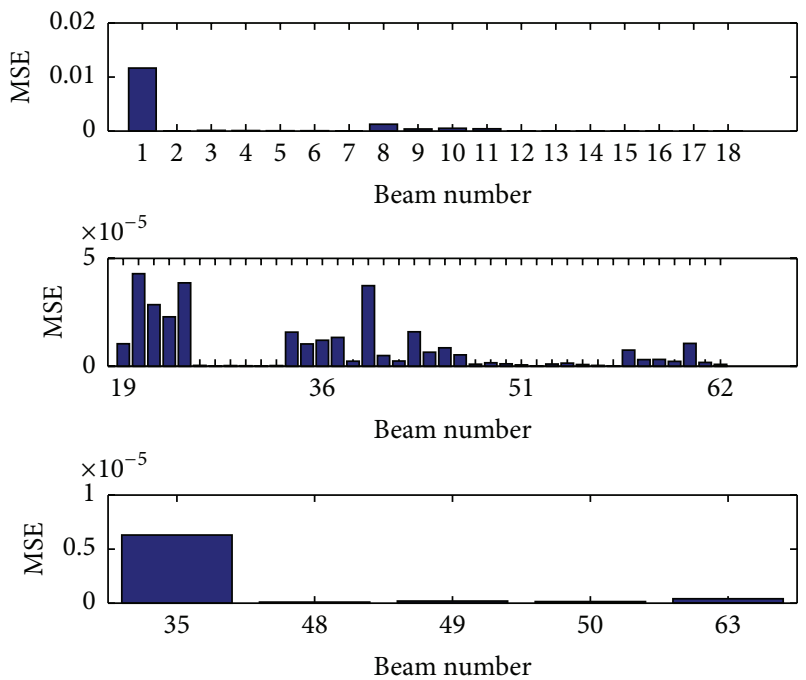

Figure 6: Fourth-order MSE.

the most sensitive beam element. In addition, compared with the other location on the 10th or 11th beam element, location approaching node 10 has larger vibration amplitude which is appropriate location for pasting PZT. Similarly, Table 4 indicates that 19th or 37th beam element is the most sensitive beam element in $N_{b}$ while the 19 th beam element is selected. Furthermore, considering that $x$-direction vibration amplitude nearing node 19 is larger than the other position on 19th beam element while $y$-direction vibration amplitude on any location of 19th beam element is nearly the same, so position approaching node 19 on 19th beam element is another appropriate location for pasting PZT. Lastly, 35th 
TABLE 4: $y$-direction maximum amplitude of beams in $N_{b}$.

\begin{tabular}{|c|c|c|c|c|c|c|c|c|}
\hline Beam number & 19 & 20 & 23 & 25 & 28 & 34 & 36 & 37 \\
\hline Amplitude (mm) & 0.1909 & 0.0930 & 0.0423 & 0.0713 & 0.0713 & 0.1909 & 0.0930 & 0.1909 \\
\hline
\end{tabular}

TABLE 5: $y$-direction maximum amplitude of beams in $N_{h}$.

\begin{tabular}{lccccc}
\hline Beam number & 35 & 48 & 49 & 50 & 63 \\
\hline Maximum amplitude $(\mathrm{mm})$ & 0.1909 & 0.1000 & 0.0703 & 0.0135 & 0.0714 \\
\hline
\end{tabular}

TABLE 6: Maximum amplitude on 35th beam element.

\begin{tabular}{lccc}
\hline$s(\mathrm{~m})$ & $x(\mathrm{~mm})$ & $y(\mathrm{~mm})$ & $z(\mathrm{~mm})$ \\
\hline 0 & 0.00022 & 0.19 & 0.00048 \\
0.2 & 0.02 & 0.19 & 0.018 \\
0.4 & 0.003 & 0.19 & 0.025 \\
0.6 & 0.018 & 0.19 & 0.018 \\
0.8 & 0.00018 & 0.19 & 0.00038 \\
\hline
\end{tabular}

beam element is the most sensitive beam element in $N_{h}$ from Table 5. Since any location's $y$-direction vibration amplitude of 35 th beam element has nearly the same value, $z$-direction vibration amplitude is considered. Define variable $s$ as the distance between any location on 35th beam element and node 19. Table 6 shows that the middle location with the maximum vibration amplitude is another appropriate location to paste PZT.

In a word, three optimization positions are middle position on 35th beam element (1st controller CMKTC1), location approaching node 19 on 19th beam element (2nd controller CMKTC2), and location approaching node 10 on 10 th beam element (3rd controller CMKTC3).

\section{Numerical Simulation}

5.1. Characteristic Model Verification Simulation. To verify that characteristic model can accurately describe dynamic characters of system, with the literature [16], four forms of control input are selected as follows:

(a) Step signal $F_{i}(k)=10$.

(b) Smooth step signal $F_{i}(k)=0.97 u(k-1)+0.3$.

(c) $10 \mathrm{~Hz}$ sinusoidal signal $F_{i}(k)=10 \sin (20 k \pi \Delta t)$.

(d) $10 \mathrm{~Hz}$ square wave signal $F_{i}(k)$ = $10 \operatorname{sign}(\sin (20 k \pi \Delta t))$.

Four different control input signals are shown in Figure 7. Control input direction is $z$-direction of node 10 ; vibration displacement output direction is selected as $y$-direction of node 19. Output estimation error $\widehat{e}(k)$ can be expressed as $\hat{\boldsymbol{e}}(k)=x_{i}(k)-\boldsymbol{\Phi}^{T}(k-1) \widehat{\boldsymbol{\vartheta}}(k)$. Sampling time $\Delta t$ is selected as $0.001 \mathrm{~s}$.

Figures 8-11 show the output estimation error with different control input. When control input is step signal or smooth step signal, output estimation error's magnitude is $10^{-7}$. If control input is sinusoidal signal or square wave signal, output estimation error's magnitude is $10^{-5}$. Therefore,

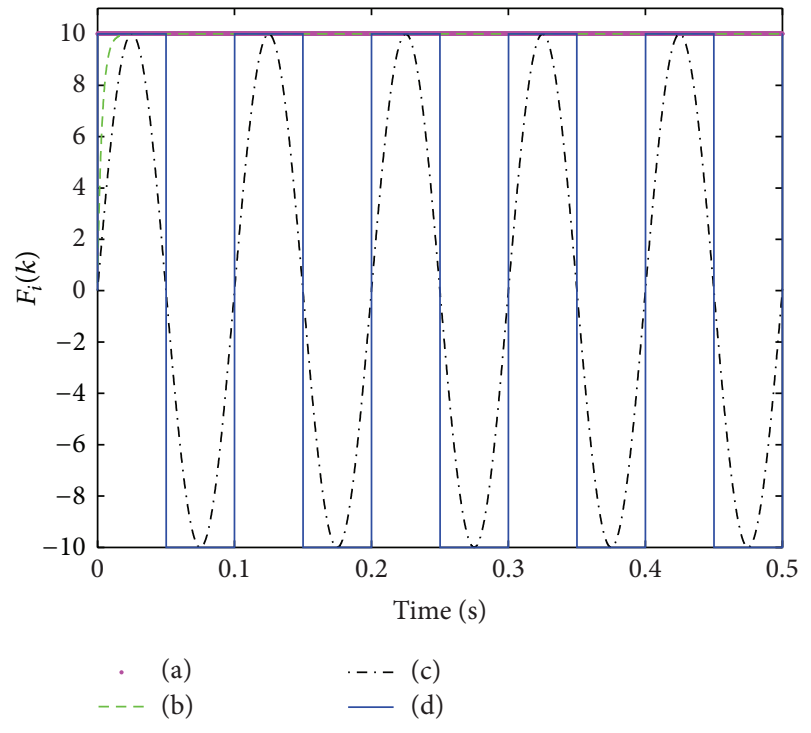

Figure 7: Four-control input signal.

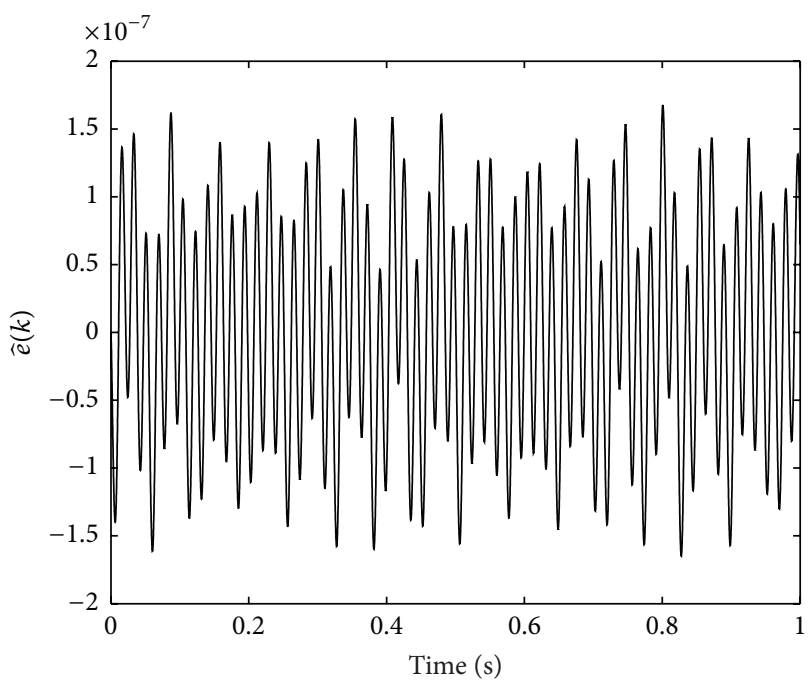

FIgURE 8: Output estimation error with (a).

output of characteristic model can approach the output of space structure. In addition, characteristic model can accurately describe space structure's dynamic characters.

5.2. Distributed PZT Vibration Simulation. Table 7 shows the related parameters of PZT and Al. With characters of characteristic parameters (9), select the initial value of 
TABLE 7: Related parameters of PZT and aluminum.

\begin{tabular}{lcccc}
\hline & Thickness $(\mathrm{mm})$ & Width $(\mathrm{mm})$ & Elastic modulus $(\mathrm{Gpa})$ & PSC $(\mathrm{C} / \mathrm{N})$ \\
\hline PZT & 1 & 10 & $8 \times 10^{10}$ & $5 \times 10^{-10}$ \\
$\mathrm{Al}$ & 5 & 12 & $7 \times 10^{10}$ & - \\
\hline
\end{tabular}

Remark 1: PSC: Piezoelectric Strain Constant.

TABLE 8: Control parameter.

\begin{tabular}{lccc}
\hline Parameter & CMKTC1 & CMKTC2 & CMKTC3 \\
\hline$\lambda$ & -0.0005 & -0.0005 & -0.025 \\
\hline
\end{tabular}

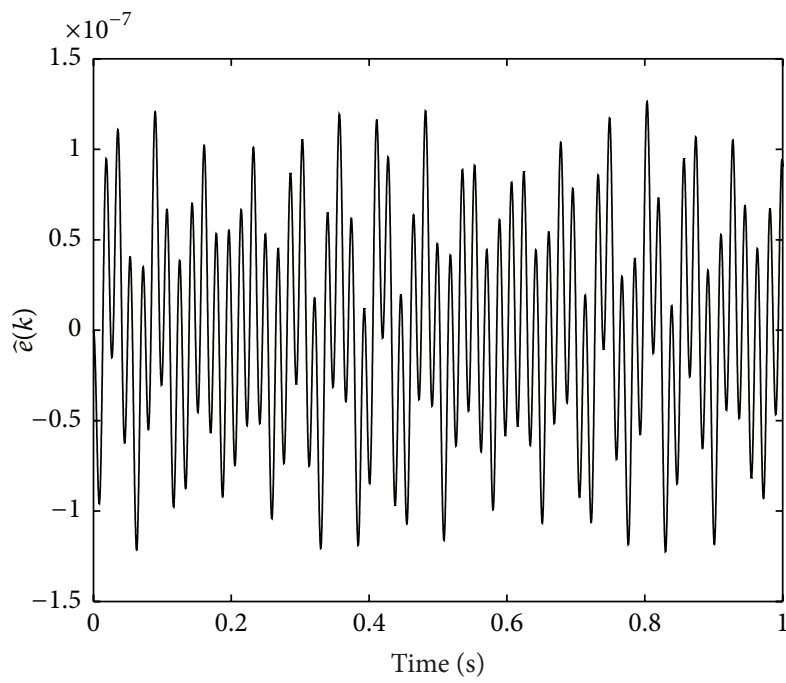

Figure 9: Output estimation error with (b).

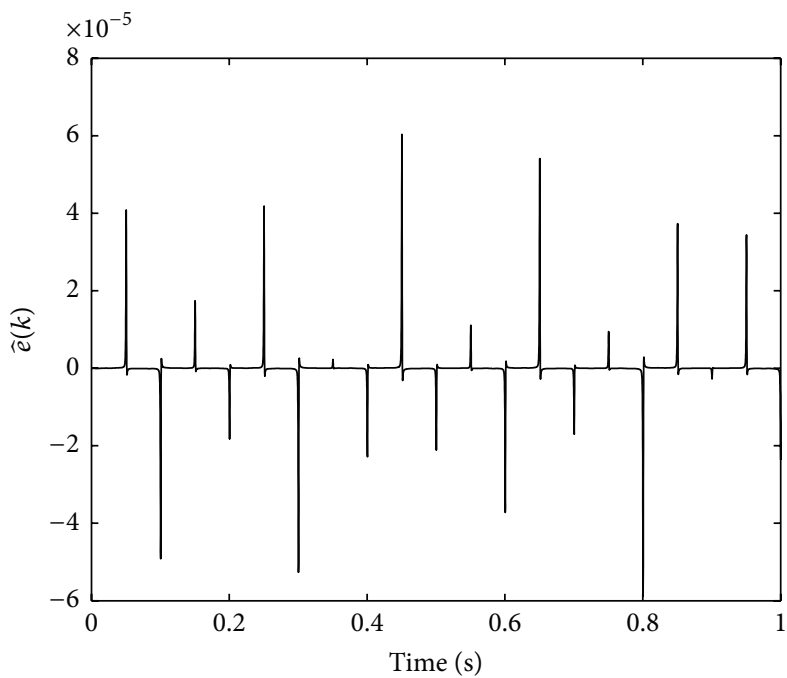

Figure 10: Output estimation error with (c).

characteristic parameters as $\widehat{\boldsymbol{\vartheta}}^{T}(0)=\left[\begin{array}{lll}1.618 & -0.618 & 0.03\end{array}\right]$. Related parameters of gradient method are $\lambda_{1}=0.8$, $\lambda_{2}=0.4$. Sampling time $\Delta t$ is selected as $0.001 \mathrm{~s}$. Control parameters are shown in Table 8. Excitation force $f_{e}=$ $10 \sin (2 \pi f t)$; excitation frequency $f$ is selected as the first natural frequency $10.03 \mathrm{~Hz}$. Select nodes 19, 22, and 25 as observation nodes. It should be emphasized that the output feedback of CMKTC1 is $y$-direction displacement.

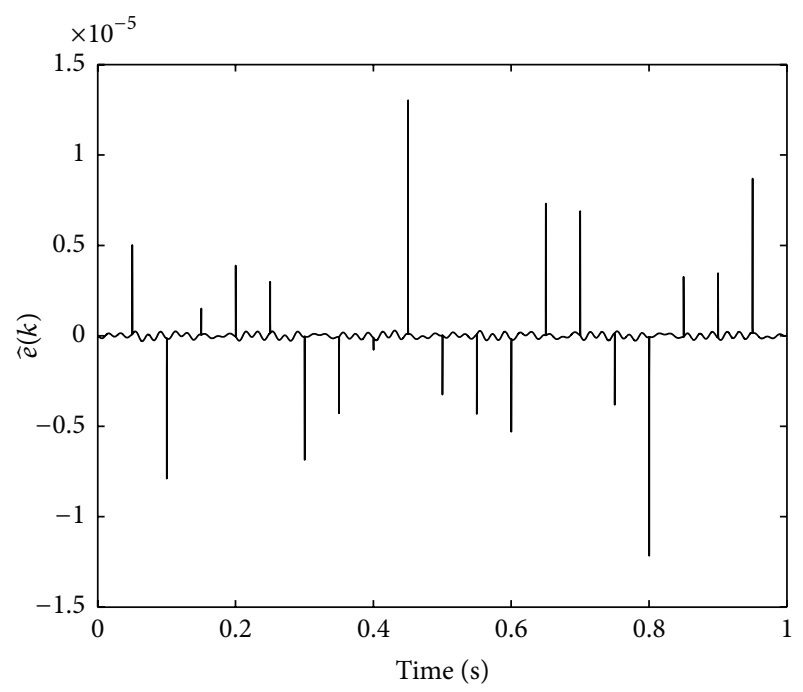

FIGURE 11: Output estimation error with (d).
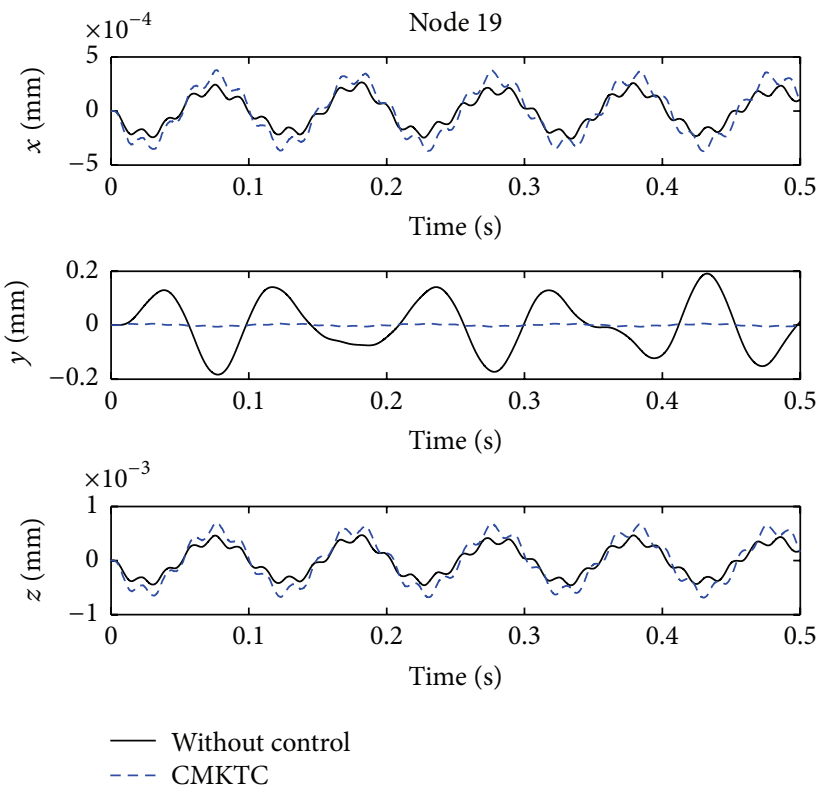

Figure 12: Displacement of node 19.

Figures 12-14 are the vibration displacement curves of nodes 19,22 , and 25 , respectively. It is shown that $y$-direction vibration displacement is much larger than $x$ or $y$ and suppressing vibration effect of $y$-direction is obvious. With CMKTC, $y$-direction vibration displacement of nodes 19,22 , and 25 attenuates to $4 \times 10^{-3}, 4.5 \times 10^{-4}$, and $1.8 \times 10^{-4}$, respectively, which indicates the effectiveness of distributed PZT vibration control. Control input is presented in Figure 15 which shows that control voltage is within voltage range of PZT. 
TABLE 9: $y$-direction vibration attenuation ratio of nodes 19, 22, and 25 with different amplitude sinusoidal disturbance.

\begin{tabular}{lccccccccccc}
\hline Disturbance amplitude & 10 & 20 & 30 & 40 & 50 & 60 & 70 & 80 & 90 & 100 \\
\hline Node 19 (\%) & 96.91 & 96.52 & 96.07 & 95.65 & 95.18 & 94.74 & 94.28 & 93.82 & 93.35 & 92.72 \\
\hline Node 22 (\%) & 96.61 & 96.71 & 96.42 & 96.16 & 95.90 & 95.45 & 95.01 & 94.54 & 94.10 & 93.65 \\
\hline Node 25 (\%) & 96.36 & 95.27 & 96.47 & 93.73 & 92.00 & 90.33 & 88.60 & 86.90 & 85.20 & 83.50 \\
\hline
\end{tabular}

TABLE 10: $y$-direction vibration attenuation ratio of nodes 19, 22, and 25 with different power-band white noise.

\begin{tabular}{lcccccccccc}
\hline Noise power & 0.05 & 0.10 & 0.15 & 0.20 & 0.25 & 0.30 & 0.35 & 0.40 & 0.45 & 0.50 \\
\hline Node 19 (\%) & 95.45 & 94.76 & 93.82 & 93.51 & 92.98 & 92.50 & 92.15 & 91.83 & 91.37 & 91.20 \\
\hline Node 22 (\%) & 96.24 & 96.34 & 96.21 & 96.21 & 96.21 & 96.21 & 96.19 & 96.18 & 96.16 & 96.15 \\
\hline Node 25 (\%) & 96.00 & 95.83 & 95.73 & 95.63 & 95.53 & 95.43 & 95.33 & 95.30 & 95.23 & 95.17 \\
\hline
\end{tabular}
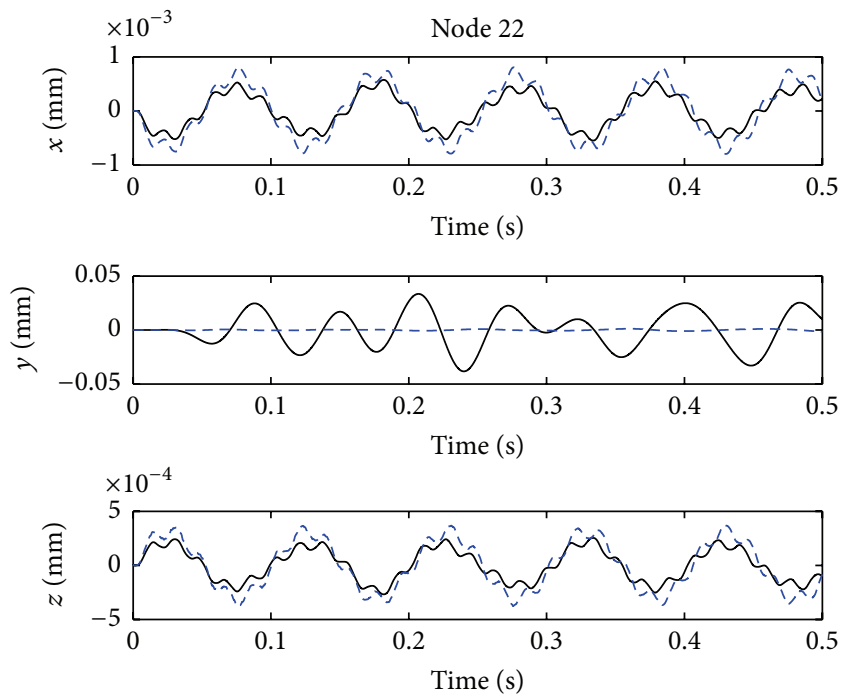

- Without control

-- CMKTC

FIGURE 13: Displacement of node 22.
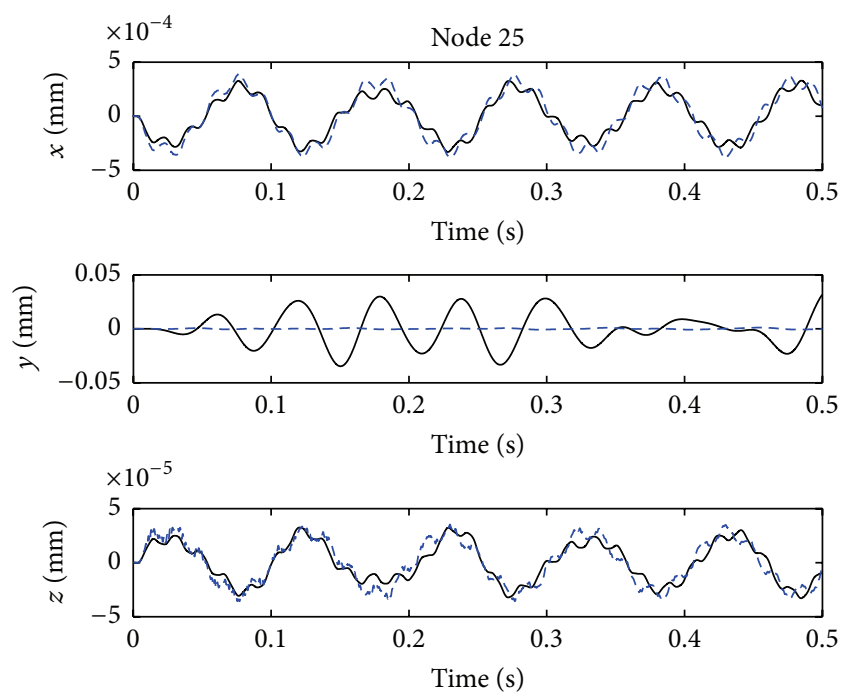

$$
\begin{aligned}
& \text { - Without control } \\
& \text {-- CMKTC }
\end{aligned}
$$

FIGURE 14: Displacement of node 25.
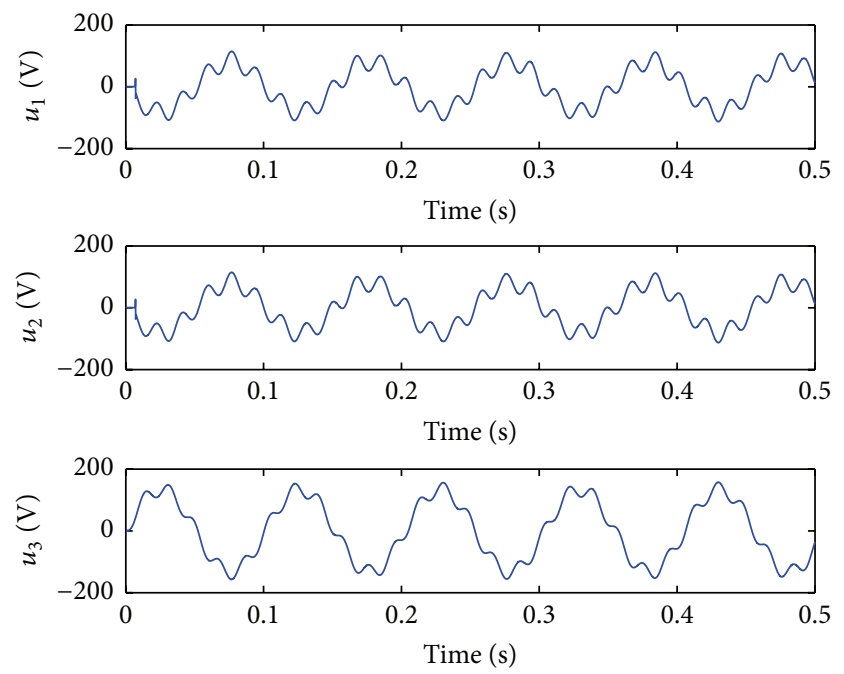

FIGURE 15: Control input.

To verify the robustness of CMKTC, the matched disturbance is added. When the sinusoidal matched disturbance $f_{e}=A \sin (200 t)$ with different amplitude is added, Table 9 shows $y$-direction vibration attenuation ratio of nodes 19 , 22 , and 25. From the table, it is known that node 25 is more sensitive than node 19 and node 22. No matter how, sinusoidal disturbance has small effect on $y$-direction vibration displacement.

Considering that there exists noise in actual system, so band-white noise with different noise power is added. $y$ direction vibration attenuation ratio of nodes 19,22 , and 25 is presented in Table 10. The table indicates that vibration damping ratio decreases with the increase of noise power. Of course, band-white noise has small effect on system performance and CMKTC is with strong robustness.

\section{Conclusion}

In this paper, a novel distributed PZT control strategy based on characteristic model has been proposed. To avoid the difficulty in obtaining the mechanical model of space structure, an efficient model-free modeling approach, that is, the so-called characteristic model theory, is adopted. 
Then a PZT position optimization method based on MSE and maximum vibration amplitude has been presented, in which the placement optimization of sensors and actuators is realized. Due to the established characteristic model, a simple but efficient CMKTC is designed. The power of the proposed control strategy is demonstrated on several numerical examples.

\section{Conflict of Interests}

The authors declare that there is no conflict of interests regarding the publication of this paper.

\section{Acknowledgment}

This work was supported by the National Natural Science Foundation of China (no. 61573332).

\section{References}

[1] G. L. de Abreu, G. P. de Melo, V. Lopes, and M. J. Brennan, "Active modal damping control of a smart truss structure using a self-organizing fuzzy controller," Journal of the Brazilian Society of Mechanical Sciences and Engineering, vol. 37, no. 2, pp. 441-450, 2015.

[2] G. Zhao, J. Wang, and Y. Gu, "Optimal placement of piezoelectric active bars in vibration control by topological optimization," Acta Mechanica Sinica, vol. 24, no. 6, pp. 699-708, 2008.

[3] B. Jaishi and W.-X. Ren, "Structural finite element model updating using ambient vibration test results," Journal of Structural Engineering, vol. 131, no. 4, pp. 617-628, 2005.

[4] B. Xu and J. S. Jiang, "Integrated optimization of structure and control for piezoelectric intelligent trusses with uncertain placement of actuators and sensors," Computational Mechanics, vol. 33, no. 5, pp. 406-412, 2004.

[5] J.-S. Hwang, A. Kareem, and W.-J. Kim, "Estimation of modal loads using structural response," Journal of Sound and Vibration, vol. 326, no. 3-5, pp. 522-539, 2009.

[6] B. Hazra, A. Sadhu, A. J. Roffel, and S. Narasimhan, "Hybrid time-frequency blind source separation towards ambient system identification of structures," Computer-Aided Civil and Infrastructure Engineering, vol. 27, no. 5, pp. 314-332, 2012.

[7] M. P. Schoen, R. C. Hoover, S. Chinvorarat, and G. M. Schoen, "System identification and robust controller design using genetic algorithms for flexible space structures," Journal of Dynamic Systems, Measurement and Control, vol. 131, no. 3, pp. 1-10, 2009.

[8] S. H. Chen, Z. J. Cao, and Z. S. Liu, "A method of for the section of sensors and actuators location," Acta Mechanica Sinica, vol. 14, no. 4, pp. 353-362, 1998.

[9] W. P. Li and H. Huang, "Integrated optimization of actuator placement and vibration control for piezoelectric adaptive trusses," Journal of Sound and Vibration, vol. 332, no. 1, pp. 17-32, 2013.

[10] Y. Luo, M. Xu, B. Yan, and X. Zhang, "PD control for vibration attenuation in Hoop truss structure based on a novel piezoelectric bending actuator," Journal of Sound and Vibration, vol. 339, pp. 11-24, 2015.
[11] G. Abreu, M. Brennan, and J. Lopes, "Robust control of an intelligent truss structure," in Proceedings of the 10th International Conference on Recent Advances in Structural Dynamics, Southampton, UK, July 2010.

[12] J. Y. Yang, Z. Q. Liu, X. Cui et al., "Experimental study of adaptive fuzzy sliding mode control for vibration of a flexible rectangular plate," International Journal of Aeronautical and Space Sciences, vol. 16, no. 1, pp. 28-40, 2015.

[13] J. Lin and Y. B. Zheng, "Vibration suppression control of smart piezoelectric rotating truss structure by parallel neuro-fuzzy control with genetic algorithm tuning," Journal of Sound and Vibration, vol. 331, no. 16, pp. 3677-3694, 2012.

[14] S. N. Mahmoodi and M. Ahmadian, "Active vibration control with modified positive position feedback," Journal of Dynamic Systems, Measurement and Control, vol. 131, no. 4, 8 pages, 2009.

[15] J. Wilhelm and R. Rajamani, "Methods for multimodal vibration suppression and energy harvesting using piezoelectric actuators," Journal of Vibration and Acoustics, vol. 131, no. 1, pp. 273-283, 2009.

[16] H. X. Wu, J. Hu, and Y. C. Xie, Characteristic Model-Based Intelligent Adaptive Control, Science and Technology Press of China, Beijing, China, 2009.

[17] H. X. Wu, J. Hu, and Y. C. Xie, "Characteristic model-based all-coefficient adaptive control method and its applications," IEEE Transactions on Systems, Man and Cybernetics Part C: Applications and Reviews, vol. 37, no. 2, pp. 213-221, 2007.

[18] A. Preumont, "On the damping of a piezoelectric truss," in Proceedings of the 21st International Congress of Theoretical and Applied Mechanics, pp. 287-301, Warsaw, Poland, August 2005. 


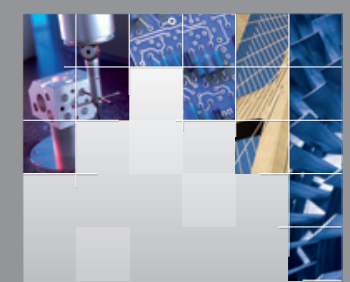

\section{Enfincering}
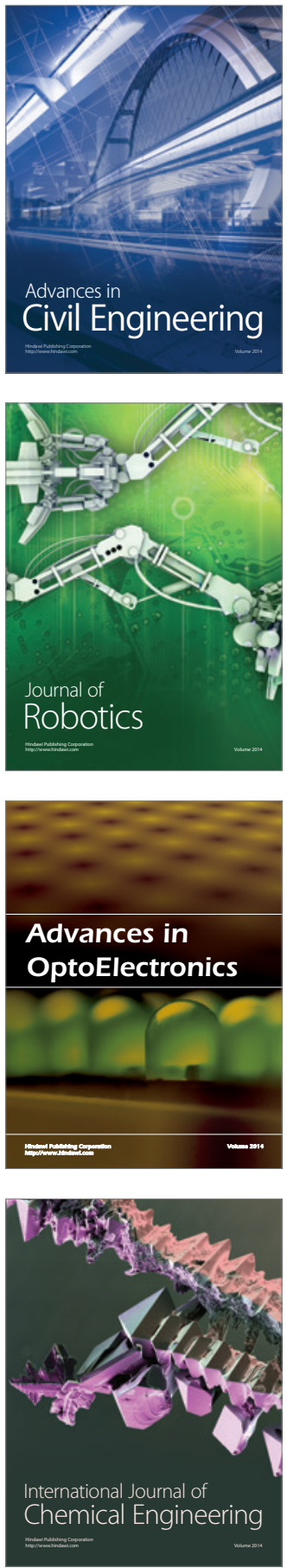

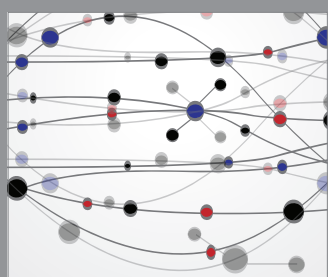

The Scientific World Journal

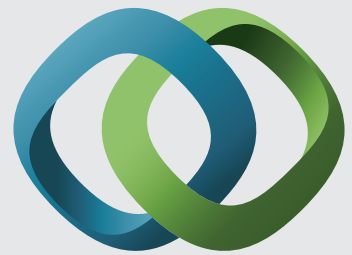

\section{Hindawi}

Submit your manuscripts at

http://www.hindawi.com
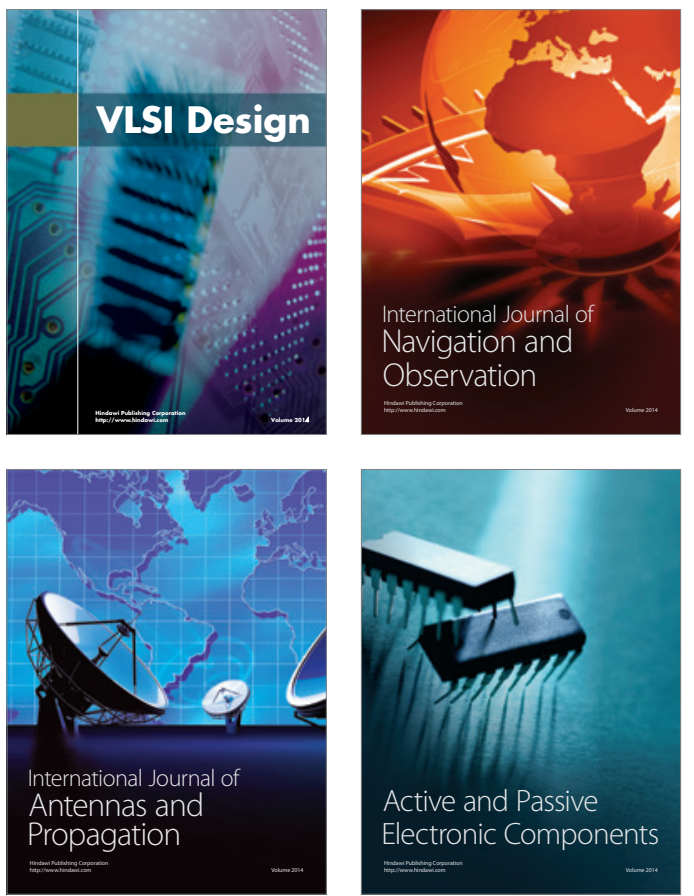
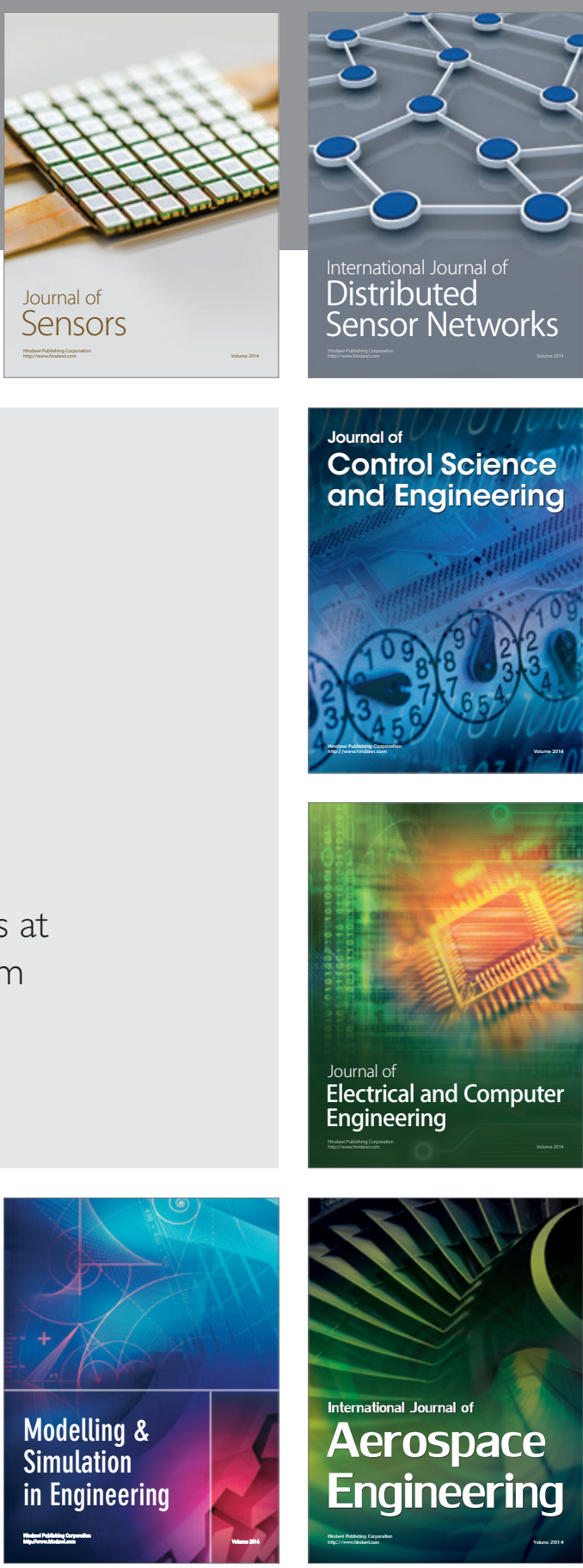

International Journal of

Distributed

Sensor Networks

Journal of

Control Science

and Engineering
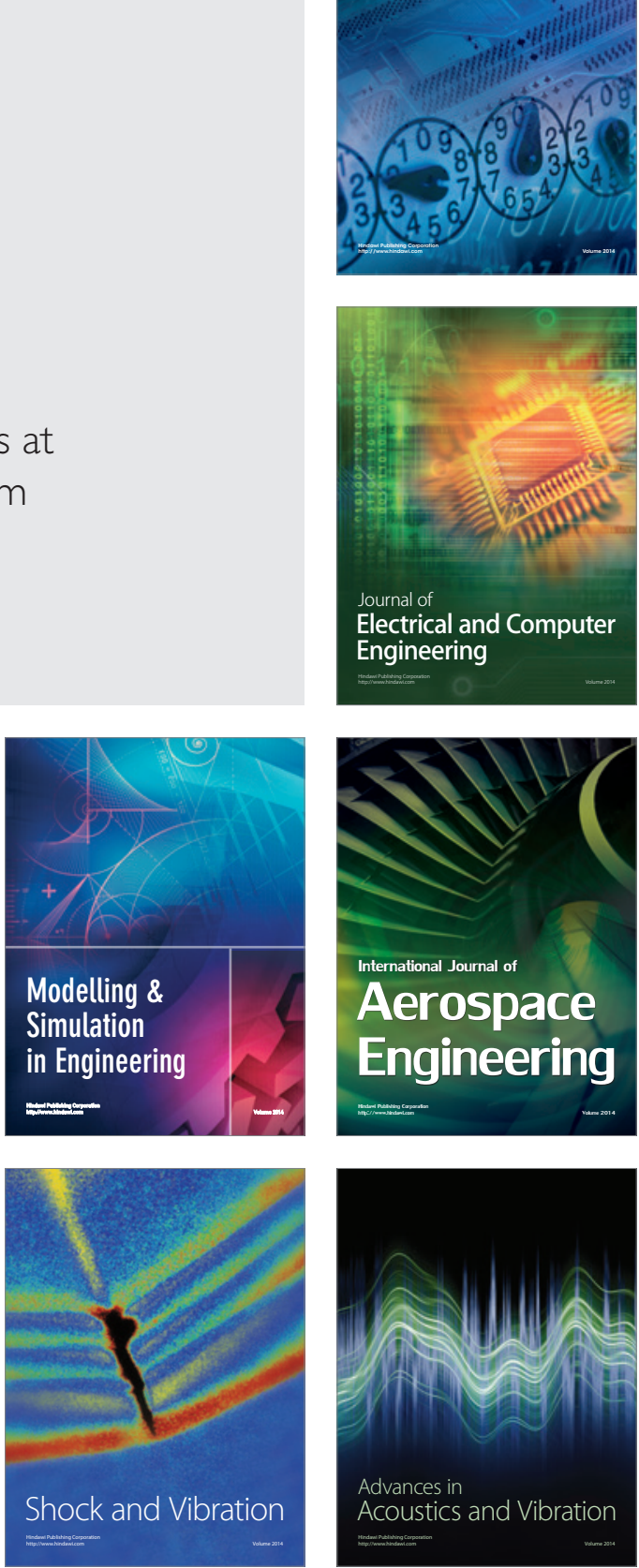